\title{
Sarcopenia and myosteatosis at presentation adversely affect survival after esophagectomy for esophageal cancer
}

\author{
Matevz Srpcic ${ }^{1,2}$, Taja Jordan ${ }^{3}$, Karteek Popuri ${ }^{4}$, Mihael Sok ${ }^{1,2}$ \\ ${ }^{1}$ Department of thoracic surgery, Surgical clinic, University Medical Centre Ljubljana, Slovenia \\ 2 Faculty of Medicine, University of Ljubljana, Slovenia \\ ${ }^{3}$ Institute of radiology, University Medical Centre Ljubljana, Slovenia \\ ${ }^{4}$ Simon Fraser University, Burnaby, Canada
}

Radiol Oncol 2020; 54(2): 237-246.

Received 7 January 2020

Accepted 3 March 2020

Correspondence to: Matevž Srpčič, M.D., Department of Thoracic Surgery, Surgical Clinic, University Medical Centre Ljubljana, Zaloška 7, SI-1000 Ljubljana, Slovenia. Phone: +386 1522 3813; fax +386 1522 2485; E-mail: matevz.srpcic@kclj.si

Disclosure: MSr, TJ and MSo declare that they have no competing interests. KP is a co-founder of and actively directs Voronoi Health Analytics Incorporated, a Canadian corporation that sells commercial licenses for the ABACS (Automated Body Composition Analyzer using Computed tomography image Segmentation) software.

Background. Esophageal cancer remains a disease with poor survival and many complications. Measuring muscle mass and quality can identify patients with diminished muscle mass (sarcopenia) and muscle fat infiltration (myosteatosis). We studied the impact of sarcopenia and myosteatosis in resectable esophageal cancer on overall survival and complications.

Patients and methods. 139 patients received a radical esophagectomy. Skeletal muscle area (SMA) and muscle attenuation (MA) in CT images at $\mathrm{L} 3$ level were recorded and groups with and without sarcopenia and myosteatosis were compared for overall survival (OS), perioperative mortality, conduit complications, pleuropulmonary complications, respiratory failure requiring mechanical ventilation and other significant complications.

Results. Prevalence of sarcopenia and myosteatosis at presentation was $16.5 \%$ and $51.8 \%$, respectively. Both were associated with decreased OS. Median survival was 18.3 months ( $\mathrm{Cl}$ 5.4-31.1) vs. 31.0 months (Cl 7.4-54.6) for sarcopenia/no sarcopenia (log rank $\mathrm{p}=0.042)$ and 19.0 months $(\mathrm{Cl} 13.3-24.7)$ vs. 57.1 months $(\mathrm{Cl} 15.2-99.0)$ for myosteatosis ( $\log$ rank $p=0.044$ ), respectively. A relationship between sarcopenia and myosteatosis and other negative outcomes after esophagectomy could not be established.

Conclusions. Sarcopenia and myosteatosis before esophagectomy are associated with decreased overall survival but not with more frequent perioperative complications. Identification of patients at risk can guide therapeutic decisions and interventions aimed at replenishing muscle reserves.

Key words: sarcopenia; myosteatosis; esophagectomy; survival; esophageal cancer; muscle depletion

\section{Introduction}

Constant gradual improvements of operative techniques and perioperative care have reduced the dangers of esophagectomy, the cornerstone of radical treatment of resectable esophageal cancer, but it remains a major procedure burdened with high morbidity and mortality. ${ }^{1}$ Overall 5-year survival in resectable esophageal cancer has improved in recent years by about $2-3$ fold. ${ }^{2}$ This improvement was attributed to centralization of surgical treatment and introduction of neoadjuvant chemoradiotherapy. ${ }^{3}$ Advances were also made in perioperative care and better understanding and prevention of the detrimental effects of muscle depletion so typical of esophageal malignancies. ${ }^{4}$

Further improvement in outcomes can be achieved by tailoring the treatment to patients' ability to withstand the trauma of surgery and to return to a functional life after treatment. Adequate 
fitness for treatment has traditionally been assessed from various performance scores, risk scores as well as more basic patients' characteristics like age and body mass index. ${ }^{5}$ Body mass index (BMI) at presentation has proven to be an inaccurate predictor of outcomes since it does not correspond to body composition well. ${ }^{6,7}$ Better methods to assess the most important parameter of body composition, the skeletal muscle content, have been introduced. They include functional tests like muscle strength measurements and measurements of muscle mass with dual energy x-ray imaging (DEXA), bioimpedance analysis or cross-sectional imaging (CT or MRI). ${ }^{8}$ Cross-sectional imaging (or planimetry) has the advantage of being readily available in cancer patients for staging purposes. This has encouraged many studies to examine the relationship between overall muscle mass, its quality and their effect on outcomes. A reliable relationship between planimetrically determined muscle mass and quality and its function, determined by other methods available, has been established. Muscle area the level of $3^{\text {rd }}$ lumbal vertebra, normalized for height (skeletal muscle index (SMI)) is highly correlated with total body skeletal muscle mass. ${ }^{9}$

Estimating survival chances for a patient presenting with resectable esophageal cancer is important in planning appropriate treatment strategies and interventions aimed at improving survival and quality of life. Pronounced weight loss is a hallmark of malignant disease, especially pronounced in digestive tract tumors, among them in esophageal and pancreatic cancers in particular. ${ }^{10}$ In their seminal work, the team from University of Alberta have shown that skeletal muscle depletion (sarcopenia and low muscle attenuation) is the real negative predictor of survival regardless of overall body weight in cancer patients. ${ }^{6}$

Sarcopenia is defined by the European Working Group on Sarcopenia in Older people as the presence of low muscle mass (under the 5th percentile) and low muscle function (strength or performance) ${ }^{11}$ typically presenting in advanced age but also in cancer and other diseases. It is a well established predictor of poor survival and treatment outcomes in cancer patients. ${ }^{6}$ Myosteatosis is defined as abnormal fat infiltration in skeletal muscle. It is negatively associated with muscle strength and quality and is brought on by aging ${ }^{12}$, diabetes ${ }^{13}$, obesity ${ }^{14}$ and malignant disease. ${ }^{6,15,16}$ Radiodensity of human muscle on CT scan (or muscle attenuation, MA) correlates well with its triglyceride content. ${ }^{14}$ Measuring the attenuation values of muscle tissue corresponds well to the extent of myosteato- sis, which is a sign of muscle wasting and again a predictor of poor outcome. ${ }^{17}$

By assessing muscle mass and quality before treatment an individualized risk assessment for overall survival and complications during treatment can be improved, patients at risk identified and appropriate interventions (mainly directed towards maintaining and gaining muscle mass) undertaken. ${ }^{18}$ Our aim was to study the impact of muscle depletion (sarcopenia and myosteatosis) on outcomes (overall survival [OS], perioperative mortality and rate of complications) in resectable esophageal cancer.

\section{Patients and methods}

\section{Study population}

All patients who received an esophagectomy with curative intent for esophageal or esophago-gastric junction cancer at Clinical Department of Thoracic Surgery at University Medical Centre Ljubljana were eligible for inclusion in the study. Patients received either upfront surgery or neoadjuvant chemoradiotherapy followed by esophagectomy according to national guidelines. All patients received individualized nutritional support and counselling according to ESPEN best practice guidelines ${ }^{19}$ and in all patients a catheter feeding jejunostomy was placed during esophagectomy. Clinical parameters were recorded prospectively in a database since 2003. Out of the 162 patients operated on consecutively between 2008 and 2018 CT images suitable for analysis of muscle mass and quality were available for 139 patients which were included in the study. Requirements for adequate images were the inclusion of L3 level and availability of non-contrast images for attenuation analysis. Only images recorded at presentation before the initiation of any treatment were considered.

Our study design was approved and the need for obtaining informed consent from participants waived by the Slovenian National medical ethics committee (approval number 0120-301/2016-2).

\section{Definitions}

We grouped complications into following groups. Conduit complications included clinically silent fistulae seen on esophagograms and/or CT scans, clinically important leaks that required interventions and frank gastric necroses. Respiratory complications included respiratory failure requiring mechanical ventilation and pneumonia, defined as the 
presence of new infiltrates on chest radiography and a positive culture result from bronchoalveolar lavage or sputum requiring antibiotics. Respiratory failure requiring mechanical ventilation was recorded separately as well.

Other complications were defined as other serious complications (Dindo Clavien 2 or greater) ${ }^{20}$ requiring intervention (i.e. early reoperation, cardioversion, endoscopic intervention) or directoscopically proven laryngeal nerve paralysis. ${ }^{21}$

OS was defined as the time interval between esophagectomy and death of any cause. Patients alive on 1.10.2018 as reported by Cancer registry of Slovenia were censored at that date.

$B M I$ was calculated as patient weight $[\mathrm{kg}] /$ height $[\mathrm{m}]^{2}$, recorded at admission one day before surgery.

\section{CT body composition analysis (planimetry)}

Pre-operative abdominal CT or whole body PETCT scans were obtained. In each patient a single slice at the level of the 3rd lumbar vertebra (L3) was selected for automatic segmentation. CT scans were analyzed using the "Automated Body Composition Analyzer using Computed tomography image Segmentation" (ABACS) software 22,23 , which uses a priori information about the skeletal muscle shape in the L3 region and predefined Hounsfield units (HU) values to recognize different tissues. HU values used to assess the total cross-sectional area for muscular tissue (SMA - skeletal muscle area) were -29 to $+150 \mathrm{HU}$. Muscle attenuation (MA) was assessed by averaging HU of skeletal muscle. Additionally, SMI was calculated using the following formula: (SMA [cm2])/(patient height [m2]). All abdominal CT and PET-CT scans were analyzed by one blinded independent radiologist.

The following planimetry data were reported: number of days between CT and esophagectomy, SMA (skeletal muscle area) reported in $\mathrm{cm}^{2}$, SMI (skeletal muscle index) is SMA corrected for height (i.e. divided by height squared) and expressed in $\mathrm{cm}^{2} / \mathrm{m}^{2}$. MA (muscle attenuation) was reported in Hounsfield units.

Previously defined muscle index cut-off values for sarcopenia in a healthy non-elderly Caucasian population were used to define limits for SMI in men at less than $43.1 \mathrm{~cm}^{2} / \mathrm{m}^{2}$ and less than $32.7 \mathrm{~cm}^{2}$ / $\mathrm{m}^{2}$ in women. Cutoff values for myosteatosis from the same study were used with myosteatosis defined as MA of less than $30.9 \mathrm{HU}$ in men and 24.8 $\mathrm{HU}$ in women. ${ }^{24}$

\section{Outcomes and statistical analysis}

Standard descriptive statistics of demographic and clinical characteristics for patients with and without sarcopenia and myosteatosis were summarized. Differences in demographic and clinical characteristics between groups (sarcopenia/no sarcopenia and myosteatosis/no myosteatosis) were evaluated with Pearson's Chi-square tests for categorical and t-tests for parametric variables.

Primary outcome studied was overall survival. It was reported in each group with the KaplanMeier curve and the survival of groups with/without sarcopenia and with/without myosteatosis was compared using the log rank Mantel Cox test.

Secondary outcomes of interest were the incidences of complications in groups with/without sarcopenia and with/without myosteatosis. They were compared with Pearson's Chi-square test. P value of $<0.05$ was considered significant. All statistical analyses were performed using Statistical Package for the Social Sciences (SPSS, version 22.0, Armonk NY).

\section{Results}

\section{Patient characterictics}

One hundred and thirty-nine patients underwent esophagectomy with primary reconstruction with curative intent. Overall demographic, clinical and complication characterictics are summarized in Table 1. Mean BMI was $26.3 \pm 4.8$ with only $7(5.0 \%)$ having a BMI less than 18.5. As many as $46(33.1 \%)$ patients reported having lost $10 \%$ or more of their normal body weight prior to esophagectomy. Average time between CT and esophagectomy was $76.9 \pm 52.3$ days with a much shorter time in those receiving primary resection compared to those with neoadjuvant treatment. Sarcopenia was present in $23(16.5 \%)$ patients and myosteatosis in $72(51.8 \%)$.

\section{Surgery and pathology}

Eighty-seven (62.6\%) patients received an open esophagectomy and $52(37.4 \%)$ had a hybrid or completely minimally invasive procedure. Type of procedure data, radicality rates, numbers of lymph nodes harvested and histology and staging data are given in Table 1.

\section{Complications and survival}

9 patients died after esophagectomy during the initial hospitalization (in hospital mortality of $6.5 \%$ ). 
TABLE 1. Demographic, preoperative, procedure and outcome data in all patients $(\mathrm{N}=139)$

\begin{tabular}{|c|c|}
\hline \multicolumn{2}{|l|}{ Demographic and preoperative data } \\
\hline Age at Surgery (mean \pm SD) [years] & $63.9 \pm 9.5$ \\
\hline $\min -\max$ & $30-83$ \\
\hline Gender (N, \% female) & $22(15.8 \%)$ \\
\hline BMI (mean $\pm S D$ ) $\left[\mathrm{kg} / \mathrm{m}^{2}\right]$ & $26.3 \pm 4.8$ \\
\hline Weight loss > 10\% (N, \%) & $46(33.1 \%)$ \\
\hline Neoadjuvant therapy (N, \%) & $74(53.2 \%)$ \\
\hline \multicolumn{2}{|l|}{ Planimetry data } \\
\hline \multicolumn{2}{|l|}{ Days between CT and esophagectomy } \\
\hline all (mean $\pm S D$ ) & $76.9 \pm 52.3$ \\
\hline $\min -\max$ & $6-192$ \\
\hline median & 84 \\
\hline Neoadjuvant (mean \pm SD) & $115.2 \pm 36.0$ \\
\hline $\min -\max$ & $14-192$ \\
\hline median & 125 \\
\hline No neoadjuvant (mean \pm SD) & $33.5 \pm 28.5$ \\
\hline $\min -\max$ & $6-141$ \\
\hline median & 23 \\
\hline \multicolumn{2}{|l|}{$\mathrm{SMA}\left[\mathrm{cm}^{2}\right]($ mean $\pm \mathrm{SD})$} \\
\hline male & $157.6 \pm 28.0$ \\
\hline female & $103.9 \pm 16.3$ \\
\hline \multicolumn{2}{|l|}{$\mathrm{SMI}\left[\mathrm{cm}^{2} / \mathrm{m}^{2}\right]($ mean $\pm \mathrm{SD})$} \\
\hline male & $52.1 \pm 9.5$ \\
\hline female & $39.8 \pm 6.8$ \\
\hline \multicolumn{2}{|l|}{ Muscle attenuation $[\mathrm{HU}]($ mean $\pm \mathrm{SD})$} \\
\hline male & $31.2 \pm 8.3$ \\
\hline female & $27.8 \pm 8.7$ \\
\hline sarcopenia $(\mathrm{N}, \%)$ & $23(16.5 \%)$ \\
\hline myosteatosis (N, \%) & $72(51.8 \%)$ \\
\hline
\end{tabular}

Almost half or 65 patients (46.8\%) experienced a complication of Dindo-Clavien grade 2 severity or greater ${ }^{20}$ after the procedure. Rates of other complications and survival rates are shown in Table 1. Survival is shown as a Kaplan-Meier curve in Figure 1. Median follow up was 18.1 months (range $0-115) .72$ patients $(51.8 \%$ ) died during the observation period and 67 (48.1\%) were censored.

\section{Sarcopenia and myosteatosis subgroups}

Demographic and clinical data was compared between patients with and without sarcopenia and with and without myosteatosis (Table 2). Patients with myosteatosis were significantly older than patients without it whereas in patients with or with-

\begin{tabular}{|c|c|}
\hline \multicolumn{2}{|l|}{ Procedure data } \\
\hline \multicolumn{2}{|l|}{ Surgical approach (N, \%) } \\
\hline open & $87(62.6 \%)$ \\
\hline MIE & $52(37.4 \%)$ \\
\hline \multicolumn{2}{|l|}{ Type of esophagectomy (N, \%) } \\
\hline Ivor-Lewis & $109(78.4 \%)$ \\
\hline McKeown & $26(18.7 \%)$ \\
\hline Transhiatal & $4(2.9 \%)$ \\
\hline \multicolumn{2}{|l|}{ Radicality (N, \%) } \\
\hline RO & $130(93.5 \%)$ \\
\hline R1 & $5(3.6 \%)$ \\
\hline R2 & $4(2.9 \%)$ \\
\hline Lymph nodes (mean $\pm S D$ ) (N, \%) & $23.4 \pm 12.3$ \\
\hline $\min -\max$ & $0-76$ \\
\hline median & 21 \\
\hline \multicolumn{2}{|l|}{ Cancer type (N, \%) } \\
\hline Adenocarcinoma & $74(53.2 \%)$ \\
\hline Squamous cell carcinoma & $64(46.0 \%)$ \\
\hline GIST & $1(0.7 \%)$ \\
\hline \multicolumn{2}{|c|}{ Pathological Stage (AJCC 2017) (N, \%) } \\
\hline I & $51(36.7 \%)$ \\
\hline$\|$ & $27(19.4 \%)$ \\
\hline III & $36(25.9 \%)$ \\
\hline IVA & $23(16.5 \%)$ \\
\hline IVB & $2(1.4 \%)$ \\
\hline \multicolumn{2}{|l|}{ Complications (N, \%) } \\
\hline In hospital mortality & $9(6.5 \%)$ \\
\hline Any complication & $65(46.8 \%)$ \\
\hline Conduit complications & $21(15.1 \%)$ \\
\hline Pleuropulmonary complications & $37(26.6 \%)$ \\
\hline Respiratory failure & $26(18.7 \%)$ \\
\hline Any other complications & $42(30.2 \%)$ \\
\hline Median survival [months] & $26.8(95 \% \mathrm{Cl} 8.1-45,7)$ \\
\hline 1 year survival & $73.7 \%$ \\
\hline 3 year survival & $45.1 \%$ \\
\hline 5 year survival & $40.3 \%$ \\
\hline
\end{tabular}

AJCC $=$ American joint committee on cancer; $\mathrm{BMI}=$ body mass index; $\mathrm{Cl}=$ confidence interval; $\mathrm{CT}=$ computed tomography; $\mathrm{GIST}=$ gastrointestinal stromal tumor; $\mathrm{HU}=$ Hounsfield units; $\mathrm{MIE}=$ minimally invasive esophagectomy; SD = standard deviation; SMA = skeletal muscle area; $\mathrm{SMI}=$ skeletal muscle index

out sarcopenia age difference didn't reach statistical significance. BMI was significantly lower in sarcopenic patients but significantly higher in patients with myosteatosis. 


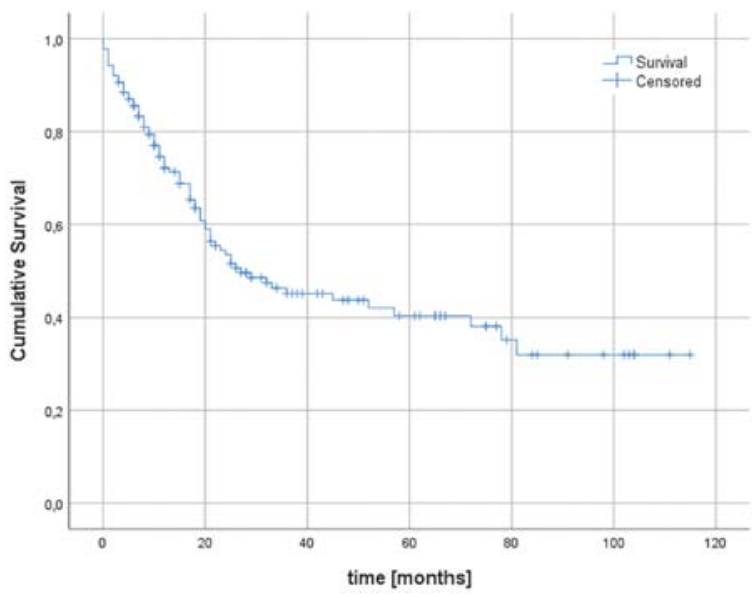

FIGURE 1. Cumulative survival Kaplan-Meier curve.

There was no statistically significant difference in sex distribution, days between CT and esophagectomy, weight loss, neoadjuvant therapy, cancer type, pathological stage, lymph nodes harvested or surgical approach between sarcopenia/ no sarcopenia and myosteatosis/no myosteatosis groups.

Complications and survival were compared between sarcopenia/no sarcopenia and myosteatosis/ no myosteatosis groups as shown in Table 3 and Figure 2 and 3.

No statistically significant difference in in hospital mortality, any complications, pleuropulmonary complications, respiratory failure or any other complications was found between sarcopenia/ no sarcopenia and myosteatosis/no myosteatosis groups. Conduit complications were however significantly less common in the myosteatosis group $(5 / 72(6.9 \%)$ vs. $16 / 67(23.9 \%)$ in patients without myosteatosis (OR 0.238 (0.082-0.692), $\mathrm{p}=0.005)$.

Survival for sarcopenia/no sarcopenia and myosteatosis/no myosteatosis is given in two Kaplan Meier plots in Figures 2 and 3. Survival curves were compared with the log rank Mantel Cox test and differences in survival between each pair were statistically significant $(p=0.042$ for sarcopenia/no sarcopenia and $p=0.044$ for myosteatosis/no myosteatosis).

\section{Discussion}

Our prospective cohort study shows that diminished muscle reserves, measured as sarcopenia (loss of muscle mass) and myosteatosis (infiltration of muscle with fat), are associated with decreased overall survival in patients receiving esophagecto-

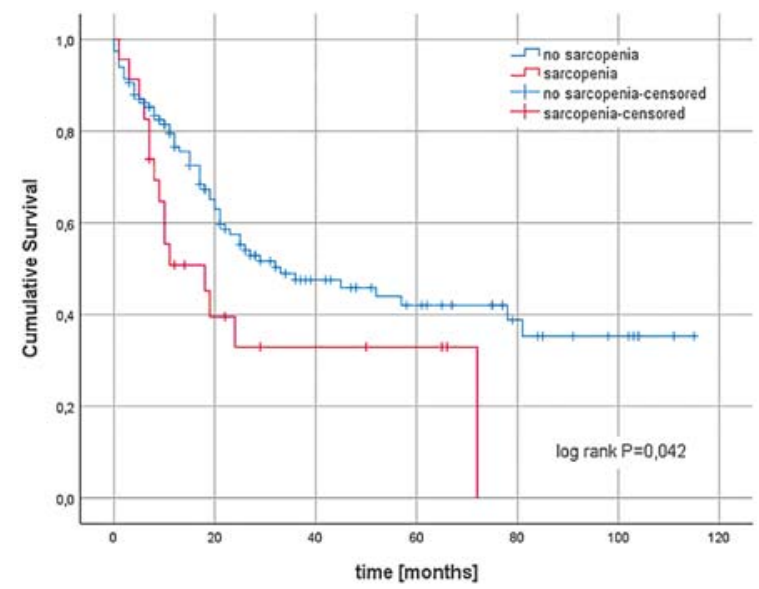

FIGURE 2. Kaplan-Meier survival curves for sarcopenia.

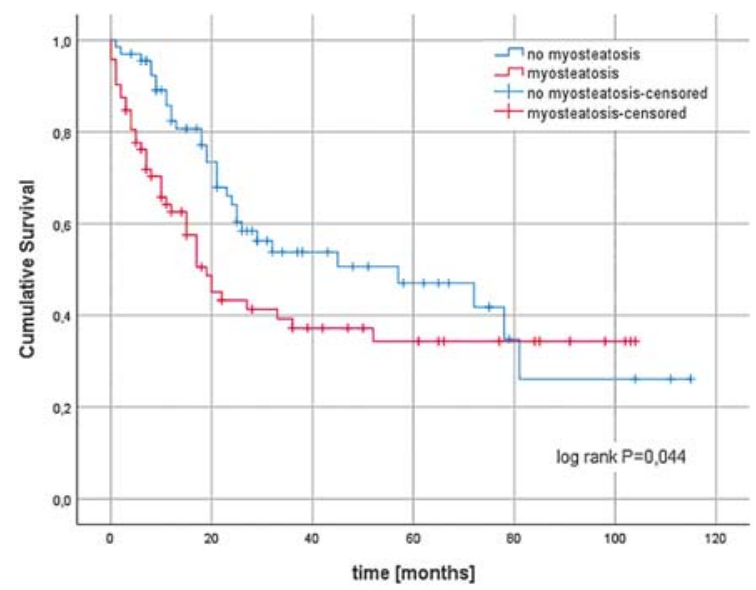

FIGURE 3. Kaplan-Meier survival curves for myosteatosis.

my as part of radical esophageal cancer treatment. A relationship between sarcopenia and myosteatosis and other negative outcomes after esophagectomy (perioperative mortality and incidence of complications) could not be established.

Effects of muscle mass loss have been studied in numerous other malignancies as well as non malignant diseases ${ }^{25-27}$ but studies reporting myosteatosis as well as sarcopenia are still rare. ${ }^{28}$ Prevalence of sarcopenia in studies on correlation between muscle area and survival in esophageal cancer can range widely from $16 \%-80 \% .{ }^{29-31}$ Choosing the right cutoff values for defining sarcopenia and myosteatosis can be challenging. In keeping with the definition of sarcopenia as absolute muscle mass below the 5 th percentile of the population ${ }^{32}$ we chose recently published cutoff values for a population closely resembling ours. Van der Werf et al. have published sex specific percentiles for SMI and MA for a healthy Caucasian population. ${ }^{24}$ They 
TABLE 2. Demographic, preoperative, pathological and procedure data compared between sarcopenia/no sarcopenia and myosteatosis/no myosteatosis groups

\begin{tabular}{|c|c|c|c|c|c|c|}
\hline & $\begin{array}{c}\text { Sarcopenia } \\
(\mathrm{N}=23(16.5 \%))\end{array}$ & $\begin{array}{l}\text { No Sarcopenia } \\
(\mathrm{N}=116(83.5 \%))\end{array}$ & $\mathrm{p}$ & $\begin{array}{l}\text { Myosteatosis } \\
(\mathrm{N}=72(51.8 \%))\end{array}$ & $\begin{array}{l}\text { No Myosteatosis } \\
(\mathrm{N}=67(48.2 \%))\end{array}$ & $\mathrm{p}$ \\
\hline Age at Surgery (mean \pm SD) & $67.1 \pm 7.8$ & $63.3 \pm 9.7$ & 0.076 & $67.1 \pm 7.7$ & $60.5 \pm 10.0$ & $<0.001$ \\
\hline Female sex (n (\%)) & $3(13.0 \%)$ & $19(16.4 \%)$ & 0.689 & $10(13.9 \%)$ & $12(17.9 \%)$ & 0.516 \\
\hline $\mathrm{BMI}($ mean $\pm S D)$ & $23.8 \pm 5.9$ & $26.7 \pm 4.4$ & 0.006 & $27.3 \pm 4.9$ & $25.2 \pm 4.4$ & 0.006 \\
\hline $\begin{array}{l}\text { Days between CT and } \\
\text { esophagectomy (mean } \pm \text { SD) }\end{array}$ & $81.4 \pm 57.6$ & $76.1 \pm 51.4$ & 0.654 & $78.8 \pm 52.8$ & $75.0 \pm 52.1$ & 0.666 \\
\hline Neoadjuvant Therapy (n (\%)) & $14(60.9 \%)$ & $60(51.7 \%)$ & 0.422 & $34(47.2 \%)$ & $40(59.7 \%)$ & 0.141 \\
\hline Cancer Type (n (\%)) & & & 0.864 & & & 0.500 \\
\hline Adenocarcinoma & $13(56.6 \%)$ & $61(52.6 \%)$ & & $37(51.4 \%)$ & $37(55.2 \%)$ & \\
\hline Squamous cell carcinoma & $10(43.4 \%)$ & $54(46.6 \%)$ & & $35(48.6 \%)$ & $29(43.3 \%)$ & \\
\hline GIST & & $1(0.8 \%)$ & & & $1(1.5 \%)$ & \\
\hline III & $4(17.4 \%)$ & $32(27.6 \%)$ & & $21(29.2 \%)$ & $15(22.4 \%)$ & \\
\hline IVA & $4(17.4 \%)$ & $19(16.4 \%)$ & & $12(16.7 \%)$ & $11(16.4 \%)$ & \\
\hline IVB & $1(4.3 \%)$ & $1(0.8 \%)$ & & $2(2.8 \%)$ & 0 & \\
\hline Lymph nodes (mean \pm SD) & $28.8 \pm 10.5$ & $23.9 \pm 12.6$ & 0.266 & $24.4 \pm 11.1$ & $22.4 \pm 13.5$ & 0.337 \\
\hline Surgical approach & & & 0.258 & & & 0.167 \\
\hline open & $12(52.2 \%)$ & 75 (64.7\%) & & $49(68.1 \%)$ & $38(56.7 \%)$ & \\
\hline MIE & $11(47.8 \%)$ & $41(35.3 \%)$ & & $23(31.9 \%)$ & $29(43.3 \%)$ & \\
\hline
\end{tabular}

$\mathrm{AJCC}=$ American joint committee on cancer; $\mathrm{BMI}=$ body mass index; $\mathrm{CT}=$ computed tomography; GIST-gastrointestinal stromal tumor; HU = Hounsfield units; MIE = minimally invasive esophagectomy; SD = standard deviation; SMA = skeletal muscle area; $S M I$ = skeletal muscle index

proposed using the 5th percentile for cutoff values for SMI and MA in non-elderly (age 20-60) to avoid age related muscle loss. These values (SMI $43.1 \mathrm{~cm}^{2} / \mathrm{m}^{2}$ for men and $32.7 \mathrm{~cm}^{2} / \mathrm{m}^{2}$ for women) are markedly lower then ones used in most previous studies. Consequently, the prevalence of sarcopenia in our study $(16.5 \%)$ is also lower than $26-75 \%$ reported in other studies in resectable esophageal cancer. Mean SMA and SMI was $157.6 \pm$ $28.0 \mathrm{~cm}^{2}$ and $52.1 \pm 9.5 \mathrm{~cm}^{2} / \mathrm{m}^{2}$ in males and $103.9 \pm$ $16.3 \mathrm{~cm}^{2}$ and $39.8 \pm 6.8 \mathrm{~cm}^{2} / \mathrm{m}^{2}$ in females (both significantly different between sexes with $p<0.001$ ) which correlates well with studies in similar populations. We believe that choosing the right population with which patients are compared is crucial in determining the real prevalence of sarcopenia (e.g., the study by Nishigori et al. in Japanese esophageal cancer patients ${ }^{33}$ used the cutoff points obtained in Canadian obese patients ${ }^{34}$ and reported sarcopenia in $75 \%$ of patients).

Defining myosteatosis is even more difficult, since the term is not used much yet and reports are scarcer. We chose cutoffs according to the same principle, i.e. at the 5 th percentile of a healthy population. We did not find a statistically significant difference in muscle attenuation between males and females $(31.2 \pm 8.3 \mathrm{HU}$ vs. $27.8 \pm 8.7 \mathrm{HU}, \mathrm{p}=$ 0.082 ), but with small numbers in our groups and the availability of sex-specific cutoff values for attenuation we opted for those. Myosteatosis was present in $51.8 \%$ of our patients and there was no significant relationship between sarcopenia and myosteatosis (OR 1.256 (CI 0.510-3.093, p = 0.620)). This is in contrast with the study by Stretch et al. where the proportions of patients with sarcopenia and myosteatosis were inverse $(40.7 \%$ vs. $25.2 \%)$ but they similarly reported no correlation between muscle mass and muscle radiodensity. A possible reason for this are the higher cutoffs they used for sarcopenia (40th percentile of their patients or 47.7 $\mathrm{cm}^{2} / \mathrm{m}^{2}$ and $\left.36.5 \mathrm{~cm}^{2} / \mathrm{m}^{2}\right){ }^{28}$

On univariate analysis sarcopenia and myosteatosis were associated with lower overall survival in our study group (Kaplan Meier log rank $\mathrm{p}=0.042$ 
TABLE 3. Complication and survival data compared between sarcopenia/no sarcopenia and myosteatosis/no myosteatosis groups

\begin{tabular}{|c|c|c|c|c|}
\hline & $\begin{array}{c}\text { Sarcopenia } \\
(\mathrm{N}=23(16.5 \%))\end{array}$ & $\begin{array}{l}\text { No Sarcopenia } \\
(\mathrm{N}=116(83.5 \%))\end{array}$ & $\begin{array}{l}\text { Odds Ratio } \\
\text { (OR. } 95 \% \mathrm{Cl} \text { ) }\end{array}$ & $\mathbf{p}$ \\
\hline \multicolumn{5}{|l|}{ Complications (n (\%)) } \\
\hline In hospital mortality & $1(4.3 \%)$ & $8(6.9 \%)$ & $0.614(0.073-5.158)$ & 0.650 \\
\hline Any complication & $11(47.8 \%)$ & $54(46.6 \%)$ & $1.052(0.430-2.578)$ & 0.911 \\
\hline Conduit complications & $4(17.4 \%)$ & $17(14.7 \%)$ & $1.226(0.371-4.049)$ & 0.738 \\
\hline Pleuropulmonary complications & $8(34.8 \%)$ & $29(25.0 \%)$ & $1.600(0.615-4.160)$ & 0.332 \\
\hline Respiratory failure & $5(21.7 \%)$ & $21(18.1 \%)$ & $1.230(0.410-3.689)$ & 0.711 \\
\hline Any other complications & $4(17.4 \%)$ & $38(32.8 \%)$ & $0.432(0.137-1.359)$ & 0.143 \\
\hline Median survival [months] & $18.3(\mathrm{Cl} 5.4-31.1)$ & $31.0(\mathrm{Cl} 7.4-54.6)$ & & 0.042 \\
\hline 1 year survival & $50.8 \%$ & $78.5 \%$ & & \\
\hline 3 year survival & $32.9 \%$ & $47.7 \%$ & & \\
\hline \multirow[t]{2}{*}{5 year survival } & $32.9 \%$ & $42.2 \%$ & & \\
\hline & $\begin{array}{c}\text { myosteatosis } \\
(\mathrm{N}=72(51.8 \%))\end{array}$ & $\begin{array}{l}\text { no myosteatosis } \\
(\mathrm{N}=67(48.2 \%))\end{array}$ & $\begin{array}{l}\text { odds ratio } \\
\text { (OR. } 95 \% \mathrm{Cl} \text { ) }\end{array}$ & $\mathbf{p}$ \\
\hline \multicolumn{5}{|l|}{ Complications (n (\%)) } \\
\hline In hospital mortality & $7(9.7 \%)$ & $2(3.0 \%)$ & $3.500(0.701-17.486)$ & 0.107 \\
\hline Any complication & $32(44.4 \%)$ & $33(49.3 \%)$ & $0.824(0.423-1.607)$ & 0.570 \\
\hline Conduit complications & $5(6.9 \%)$ & $16(23.9 \%)$ & $0.238(0.082-0.692)$ & 0.005 \\
\hline Pleuropulmonary complications & $17(23.6 \%)$ & $20(30.0 \%)$ & $0.726(0.341-1.545)$ & 0.406 \\
\hline Respiratory failure & $14(19.4 \%)$ & $12(17.9 \%)$ & $1.066(0.453-2.510)$ & 0.884 \\
\hline Any other complications & $24(33.3 \%)$ & $18(26.9 \%)$ & $1.361(0.656-2.822)$ & 0.407 \\
\hline Median survival [months] & $19.0(\mathrm{Cl} 13.3-24.7)$ & $57.1(\mathrm{Cl} 15.2-99.0)$ & & 0.044 \\
\hline 1 year survival & $64.2 \%$ & $84.0 \%$ & & \\
\hline 3 year survival & $36.9 \%$ & $53.7 \%$ & & \\
\hline 5 year survival & $33.9 \%$ & $46.9 \%$ & & \\
\hline
\end{tabular}

$\mathrm{Cl}=$ confidence interval; $\mathrm{OR}=$ odds ratio

and $\mathrm{p}=0.044$, respectively). For sarcopenia this is in accordance with previously published data and for myosteatosis this is one of the first published reports. Dijksterhuis et al. have published a report on body composition, survival and toxicity in advanced esophagogastric cancer patients receiving palliative chemotherapy where they used BMIspecific cutoff values to define myosteatosis $(<41$ HU in non obese $(\mathrm{BMI}<25)$ and $<33$ HU in overweight patients). Prevalence of myosteatosis in their group was $50 \%$ and they found a lower risk of grade III and IV toxicity in patients with higher muscular density but no association between sarcopenia or myosteatosis and survival was found..$^{35}$ Tamandl et al. published a study with 200 patients receiving an esophagectomy. They stratified patients in low- and high-muscle attenuation groups with a cutoff of $40 \mathrm{HU}$ in a population similar to ours. Average MA was $36 \mathrm{HU}$ (31-41) and patients with MA $<40 \mathrm{HU}$ had significantly poorer overall survival. ${ }^{36}$ The percentage of patients with MA over and under $40 \mathrm{HU}$ is not given, so we cannot compare the prevalence to our results but this definition of reduced muscle attenuation uses a cutoff considerably higher than ours.

On the other hand, a study by Gabiatti et al. in patients with locally advanced esophageal cancer receiving definitive chemoradiotherapy demonstrated favorable progression free survival and overall survival in a subgroup of patients with myosteatosis but without systemic inflammation. ${ }^{37}$

Sarcopenia has been studied extensively as a predictive factor in esophageal cancer. A recently published meta-analysis by Boshier et al. reviewed 
29 studies with 3193 patients (38\% sarcopenic) in which various methods were used to diagnose sarcopenia. ${ }^{38}$ Sarcopenic patients had more pulmonary complications and lower overall survival. A similar meta-analysis by Deng et al. reviewed 11 cohort studies including 1520 patients (52.3\% sarcopenic). Patients with sarcopenia had lower 3-year and 5-year survival after resection. ${ }^{39}$

Complications and perioperative mortality were compared in our study between sarcopenia/no sarcopenia and myosteatosis/no myosteatosis groups and no statistically significant negative effect of muscle depletion was found. This is in concordance with most other studies who failed to show a connection even in studies who showed differences in long term survival. ${ }^{29,40}$ Insufficient statistical power in most studies including ours to detect a potential difference in complication rates is no doubt a strong factor. For conduit complications however, the incidence in our cohort was significantly lower in the myosteatosis group (5/72 (6.9\%) vs. $16 / 67(23.9 \%)$ in patients without myosteatosis, (OR 0.238 (0.082-0.692), $\mathrm{p}=0.005)$. It is difficult to explain the reason for this observation. A higher BMI in patients with myosteatosis could indicate a better nutritional status at presentation. Despite the lower incidence of this dangerous complication perioperative mortality in patients with myosteatosis was not different than in patients without it.

General clinical data in our cohort does not differ significantly from similar published series in resectable esophageal cancer. Patients with myosteatosis were significantly older than patients without it $(67.1 \pm 7.7$ vs. $60.5 \pm 10.0(\mathrm{p}<0.001))$ whereas in patients with or without sarcopenia age difference didn't reach statistical significance $(67.1 \pm 7.8$ vs. $63.3 \pm 9.7(p=0.076))$. BMI was significantly lower in sarcopenic patients $(23.8 \pm 5.9$ vs. $26.7 \pm 4.4$ (p $=0.006)$ ) but significantly higher in patients with myosteatosis $(27.3 \pm 4.9$ vs. $25.2 \pm 4.4(p=0.006)) .13$ patients $(9.4 \%)$ had both sarcopenia and myosteatosis, their BMI was $25.5 \pm 6.1$ (range 18.1-37.1) $33.1 \%$ of our patients lost $10 \%$ or more of their body weight but this did not confer a greater risk of having sarcopenia (OR 2.12 (CI 0.855-5.266), p = 0.100 ) or myosteatosis (OR 1.165 (CI 0.574-2.366), p $=0.672)$. As suggested elsewhere ${ }^{28}$ sarcopenia and myosteatosis are probably two separate entities with different causes and effects reflecting different disturbances in metabolic processes.

Underlying causes of sarcopenia and myosteatosis are most likely overlapping to some extent. Possible mechanisms, through which they nega- tively affect survival, are various. Diminished food intake due to dysphagia and loss of appetite as well as a chronic inflammation state in esophageal cancer lead to sarcopenia. This in turn causes diminished mobility and rehabilitation after surgery $^{41}$, respiratory complications ${ }^{33}$, inferior wound healing ${ }^{42}$ and diminished tolerance of chemo and radiotherapy. ${ }^{35}$ Skeletal muscle has been described as an endocrine organ ${ }^{43}$ and it is the derangement of this function that is also a possible cause of inferior survival. Carefully designed studies are needed to corroborate this hypothesis.

The inclusion of myosteatosis assessment is in our opinion a strength of our study. We see that myosteatosis is more prevalent than sarcopenia and is a more sensitive marker of muscle degradation which precedes muscle mass and overall body mass loss. It is nevertheless at least as detrimental to prognosis as sarcopenia. Our study also uses recently published cut-off values that in our opinion assess the incidence of sarcopenia better than previous studies. However, this hinders the comparability of our results with others. It is not without weaknesses either. All CT images were recorded at staging with approximately half the patients going straight to resection and the other half receiving neoadjuvant treatment first. No repeat CT images were taken after neoadjuvant treatment if there were no clinical signs of progression according to our group's guidelines. The distribution of intervals from CT to esophagectomy is therefore bimodal and the planimetric data reflects patients' muscle reserves at beginning of any treatment and not necessarily at esophagectomy. This is a shortcoming when assessing the impact on perioperative mortality and complications since muscle mass loss is a well known process during neoadjuvant therapy. ${ }^{44-47}$ The large variation in times between CT and esophagectomy should in our opinion however not be regarded as a weakness when assessing the impact on overall survival of radical esophageal cancer treatment. Our study also lacks statistical power to detect a potential difference in mortality and complications, an issue that has fraught all previous studies as well. With growing numbers of cases in which CT images are available for analysis and with potential pooling of data these statistical issues can be overcome in the future.

Lastly, due to the univariate nature of our analysis no causal effect between survival and muscle depletion markers can be established, but the association shown can serve as an incentive for further research. 


\section{Conclusions}

In a prospective cohort study from a dedicated database on esophagectomies we studied the association of sarcopenia and myosteatosis with outcomes after curative esophagectomies with or without neoadjuvant chemoradiotherapy. Prevalence of sarcopenia and myosteatosis at presentation was $16.5 \%$ and $51.8 \%$, respectively. Both sarcopenia and myosteatosis were associated with decreased overall survival. For sarcopenia this is in accordance with previously published data and for myosteatosis this is one of the first published reports. Identifying novel predictors of outcomes can be beneficial for tailoring treatment options in patients with esophageal cancer as well as for planning intervention strategies targeted at improving functional body reserves.

\section{Authors' contributions}

MSr and MSo designed the study. MSr collected, analyzed and interpreted the data and was the major contributor in writing the manuscript. TJ collected the imaging data and was a minor contributor in writing the manuscript. TJ and $\mathrm{KP}$ analyzed and interpreted the imaging data. MSo designed the data collecting database. All authors read and approved the final manuscript.

\section{Acknowledgements}

The authors would like to sincerely thank Professor Vickie Baracos, Ph.D., for her invaluable advice and guidance in designing this study. Funding was provided by University Medical Centre Ljubljana, Slovenia. The funding body had no immediate role in the design of the study and collection, analysis, and interpretation of data and in writing the manuscript.

\section{References}

1. Low DE, Alderson D, Cecconello I, Chang AC, Darling GE, D'Journo XB, et al. International consensus on standardization of data collection for complications associated with esophagectomy: esophagectomy complications consensus group (ECCG). Ann Surg 2015; 262: 286-94. doi: 10.1097/ SLA.0000000000001098

2. Miller KD, Siegel RL, Lin CC, Mariotto AB, Kramer JL, Rowland JH, et al. Cancer treatment and survivorship statistics, 2016. CA Cancer J Clin 2016; 66: 271-89. doi: 10.3322/caac.21349
3. van Putten M, de Vos-Geelen J, Nieuwenhuijzen G, Siersema PD, Lemmens VEPP, Rosman C, et al. Long-term survival improvement in oesophageal cancer in the Netherlands. Eur J Cancer 2018; 94: 138-47. doi: 10.1016/j. ejca.2018.02.025

4. Soma D, Kawamura Yl, Yamashita S, Wake H, Nohara K, Yamada K, et al. Sarcopenia, the depletion of muscle mass, an independent predictor of respiratory complications after oncological esophagectomy. Dis Esophagus 2019; 32. pii: doy092. doi: 10.1093/dote/doy092

5. Markar SR, Low DE. Physiology, not chronology, dictates outcomes after esophagectomy for esophageal cancer: outcomes in patients 80 years and older. Ann Surg Oncol 2013; 20: 1020-6. doi: 10.1245/s10434-012-2703-x

6. Martin L, Birdsell L, MacDonald N, Reiman T, Clandinin MT, McCargar L, et al. Cancer cachexia in the age of obesity: skeletal muscle depletion is a powerful prognostic factor, independent of body mass index. J Clin Oncol 2013; 31: 1539-47. doi: 10.1200/JCO.2012.45.2722

7. Gonzalez MC, Correia MITD, Heymsfield SB. A requiem for BMI in the clinical setting. Curr Opin Clin Nutr Metab Care 2017; 20: 314-21. doi: 10.1097/ MCO.0000000000000395

8. Di Sebastiano KM, Mourtzakis M. A critical evaluation of body composition modalities used to assess adipose and skeletal muscle tissue in cancer. App Physiol Nutr Metab 2012; 37: 811-21. doi: 10.1139/h2012-079

9. Shen W, Punyanitya M, Wang Z, Gallagher D, St-Onge M-P, Albu J, et al. Total body skeletal muscle and adipose tissue volumes: estimation from a single abdominal cross-sectional image. J Appl Physiol Bethesda Md (1985) 2004; 97: 2333-8. doi: 10.1152/japplphysiol.00744.2004

10. Muscaritoli M, Lucia S, Farcomeni A, Lorusso V, Saracino V, Barone C, et al. Prevalence of malnutrition in patients at first medical oncology visit: the PreMiO study. Oncotarget 2017; 8: 79884-96. doi: 10.18632/oncotarget.20168

11. Cruz-Jentoft AJ, Baeyens JP, Bauer JM, Boirie Y, Cederholm T, Landi F, et al. Sarcopenia: European consensus on definition and diagnosis. Age Ageing 2010; 39: 412-23. doi: 10.1093/ageing/afq034

12. Delmonico MJ, Harris TB, Visser M, Park SW, Conroy MB, Velasquez-Mieyer $\mathrm{P}$, et al. Longitudinal study of muscle strength, quality, and adipose tissue infiltration. Am J Clin Nutr 2009; 90: 1579-85. doi: 10.3945/ajcn.2009.28047

13. Miljkovic I, Kuipers AL, Cvejkus R, Bunker CH, Patrick AL, Gordon CL, et al. Myosteatosis increases with aging and is associated with incident diabetes in African ancestry men. Obes Silver Spring Md 2016; 24: 476-82. doi: 10.1002/oby. 21328

14. Goodpaster BH, Theriault R, Watkins SC, Kelley DE. Intramuscular lipid content is increased in obesity and decreased by weight loss. Metabolism 2000; 49: 467-72. doi: 10.1016/s0026-0495(00)80010-4

15. Sabel MS, Lee J, Cai S, Englesbe MJ, Holcombe S, Wang S. Sarcopenia as a prognostic factor among patients with stage III melanoma. Ann Surg Oncol 2011; 18: 3579-85. doi: 10.1245/s10434-011-1976-9

16. Antoun S, Lanoy E, lacovelli R, Albiges-Sauvin L, Loriot $Y$, Merad-Taoufik M, et al. Skeletal muscle density predicts prognosis in patients with metastatic renal cell carcinoma treated with targeted therapies. Cancer 2013; 119: 3377-84. doi: 10.1002/cncr.28218

17. Aubrey J, Esfandiari N, Baracos VE, Buteau FA, Frenette J, Putman CT, et al. Measurement of skeletal muscle radiation attenuation and basis of its biological variation. Acta Physiol Oxf Engl 2014; 210: 489-97. doi: 10.1111/ apha.12224

18. Jordan T, Mastnak DM, Palamar N, Kozjek NR. Nutritional therapy for patients with esophageal cancer. Nutr Cancer 2018; 70: 23-9. doi: $10.1080 / 01635581.2017 .1374417$

19. Arends J, Baracos V, Bertz H, Bozzetti F, Calder PC, Deutz NEP, et al. ESPEN expert group recommendations for action against cancer-related malnutrition. Clin Nutr Edinb Scotl 2017; 36: 1187-96. doi: 10.1016/j. clnu.2017.06.017

20. Dindo D, Demartines N, Clavien P-A. Classification of surgical complications. Ann Surg 2004; 240: 205-13. doi: 10.1097/01.sla.0000133083.54934.ae

21. Seder CW, Raymond DP, Wright CD, Gaissert HA, Chang AC, Clinton S, et al. The Society of Thoracic Surgeons General Thoracic Surgery Database 2017 update on outcomes and quality. Ann Thorac Surg 2017; 103: 1378-83. doi: 10.1016/j.athoracsur.2017.02.073 
22. Popuri K, Cobzas D, Esfandiari N, Baracos V, Jägersand M. Body composition assessment in axial CT images using FEM-based automatic segmentation of skeletal muscle. IEEE Trans Med Imaging 2016; 35: 512-20. doi: 10.1109/ TMI.2015.2479252

23. Chung $\mathrm{H}$, Cobzas D, Birdsell L, Lieffers J, Baracos V. Automated segmentation of muscle and adipose tissue on CT images for human body composition analysis. Med Imaging 2009; 7261: 72610K. doi: 10.1117/12.812412

24. van der Werf A, Langius JAE, de van der Schueren MAE, Nurmohamed SA van der Pant KAMI, Blauwhoff-Buskermolen S, et al. Percentiles for skeletal muscle index, area and radiation attenuation based on computed tomography imaging in a healthy Caucasian population. Eur J Clin Nutr 2018; 72: 288-96. doi: 10.1038/s41430-017-0034-5

25. Deng H-Y, Hou L, Zha P, Huang K-L, Peng L. Sarcopenia is an independent unfavorable prognostic factor of non-small cell lung cancer after surgical resection: a comprehensive systematic review and meta-analysis. Eur J Surg Oncol 2019; 45: 728-35. doi: 10.1016/j.ejso.2018.09.026

26. Levolger S, van Vugt JLA, de Bruin RWF, IJzermans JNM. Systematic review of sarcopenia in patients operated on for gastrointestinal and hepatopancreatobiliary malignancies. Br J Surg 2015; 102: 1448-58. doi: 10.1002/bjs.9893

27. Waduud MA, Wood B, Keleabetswe P, Manning J, Linton E, Drozd M, et al. Influence of psoas muscle area on mortality following elective abdominal aortic aneurysm repair. Br J Surg 2019; 106: 367-74. doi: 10.1002/bjs.11074

28. Stretch C, Aubin J-M, Mickiewicz B, Leugner D, Al-Manasra T, Tobola E, et al. Sarcopenia and myosteatosis are accompanied by distinct biological profiles in patients with pancreatic and periampullary adenocarcinomas. PloS One 2018; 13: e0196235. doi: 10.1371/journal.pone.0196235

29. Paireder M, Asari R, Kristo I, Rieder E, Tamandl D, Ba-Ssalamah A, et al Impact of sarcopenia on outcome in patients with esophageal resection following neoadjuvant chemotherapy for esophageal cancer. Eur J Surg Oncol 2017; 43: 478-84. doi: 10.1016/j.ejso.2016.11.015

30. Elliott JA, Doyle SL, Murphy CF, King S, Guinan EM, Beddy P, et al. Sarcopenia: prevalence, and impact on operative and oncologic outcomes in the multimodal management of locally advanced esophageal cancer. Ann Surg 2017; 266: 822-30. doi: 10.1097/SLA.0000000000002398

31. Järvinen T, Ilonen I, Kauppi J, Salo J, Räsänen J. Loss of skeletal muscle mass during neoadjuvant treatments correlates with worse prognosis in esophageal cancer: a retrospective cohort study. World J Surg Oncol 2018; 16: 27. doi: 10.1186/s12957-018-1327-4

32. Fearon K, Strasser F, Anker SD, Bosaeus I, Bruera E, Fainsinger RL, et al. Definition and classification of cancer cachexia: an international consensus. Lancet Oncol 2011; 12: 489-95. doi: 10.1016/S1470-2045(10)70218-7

33. Nishigori T, Okabe H, Tanaka E, Tsunoda S, Hisamori S, Sakai Y. Sarcopenia as a predictor of pulmonary complications after esophagectomy for thoracic esophageal cancer. J Surg Oncol 2016; 113: 678-84. doi: 10.1002/jso.24214

34. Prado CMM, Lieffers JR, McCargar $\amalg$, Reiman T, Sawyer MB, Martin L, et al. Prevalence and clinical implications of sarcopenic obesity in patients with solid tumours of the respiratory and gastrointestinal tracts: a population-based study. Lancet Oncol 2008; 9: 629-35. doi: 10.1016/ S1470-2045(08)70153-0

35. Dijksterhuis WPM, Pruijt MJ, van der Woude SO, Klaassen R, Kurk SA, van Oijen MGH, et al. Association between body composition, survival, and toxicity in advanced esophagogastric cancer patients receiving palliative chemotherapy. J Cachexia Sarcopenia Muscle 2019; 10: 199-206. doi: 10.1002/jcsm.12371

36. Tamandl D, Paireder M, Asari R, Baltzer PA, Schoppmann SF, Ba-Ssalamah A. Markers of sarcopenia quantified by computed tomography predict adverse long-term outcome in patients with resected oesophageal or gastrooesophageal junction cancer. Eur Radiol 2016; 26: 1359-67. doi: 10.1007/ s00330-015-3963-1

37. Gabiatti CTB, Martins MCL, Miyazaki DL, Silva LP, Lascala F, Macedo LT, et al. Myosteatosis in a systemic inflammation-dependent manner predicts favorable survival outcomes in locally advanced esophageal cancer. Cancer Med 2019; 8: 6967-76. doi: 10.1002/cam4.2593

38. Boshier PR, Heneghan R, Markar SR, Baracos VE, Low DE. Assessment of body composition and sarcopenia in patients with esophageal cancer: a systematic review and meta-analysis. Dis Esophagus 2018; 31. doi: 10.1093/ dote/doy047
39. Deng H-Y, Zha P, Peng L, Hou L, Huang K-L, Li X-Y. Preoperative sarcopenia is a predictor of poor prognosis of esophageal cancer after esophagectomy: a comprehensive systematic review and meta-analysis. Dis Esophagus 2019; 32. doi: $10.1093 /$ dote/doy115

40. Tsukioka T, Nishiyama N, Izumi N, Mizuguchi S, Komatsu H, Okada S, et al. Sarcopenia is a novel poor prognostic factor in male patients with pathological Stage I non-small cell lung cancer. Jpn J Clin Oncol 2017; 47: 363-8. doi: 10.1093/jjco/hyx009

41. JR, Bathe OF, Fassbender K, Winget M, Baracos VE. Sarcopenia is associated with postoperative infection and delayed recovery from colorectal cancer resection surgery. Br J Cancer 2012; 107: 931-6. doi: 10.1038/bjc.2012.350

42. Achim V, Bash J, Mowery A, Guimaraes AR, Li R, Schindler J, et al. Prognostic indication of sarcopenia for wound complication after total laryngectomy. JAMA Otolaryngol Head Neck Surg 2017; 143: 1159-65. doi: 10.1001/ jamaoto.2017.0547

43. Pedersen BK, Febbraio MA. Muscles, exercise and obesity: skeletal muscle as a secretory organ. Nat Rev Endocrinol 2012; 8: 457-65. doi: 10.1038/ nrendo.2012.49

44. Guinan EM, Doyle SL, Bennett AE, O'Neill L, Gannon J, Elliott JA, et al. Sarcopenia during neoadjuvant therapy for oesophageal cancer: characterising the impact on muscle strength and physical performance. Support Care Cancer 2018; 26: 1569-76. doi: 10.1007/s00520-017-3993-0

45. Reisinger KW, Bosmans JWAM, Uittenbogaart M, Alsoumali A, Poeze $M$ Sosef $\mathrm{MN}$, et al. Loss of skeletal muscle mass during neoadjuvant chemoradiotherapy predicts postoperative mortality in esophageal cancer surgery. Ann Surg Oncol 2015; 22: 4445-52. doi: 10.1245/s10434-015-4558-4

46. Yip C, Goh V, Davies A, Gossage J, Mitchell-Hay R, Hynes O, et al. Assessment of sarcopenia and changes in body composition after neoadjuvant chemotherapy and associations with clinical outcomes in oesophageal cancer. Eur Radiol 2014; 24: 998-1005. doi: 10.1007/s00330-014-3110-4

47. Awad S, Tan BH, Cui H, Bhalla A, Fearon KCH, Parsons SL, et al. Marked changes in body composition following neoadjuvant chemotherapy for oesophagogastric cancer. Clin Nutr Edinb Scot/ 2012; 31: 74-7. doi: 10.1016/j. clnu.2011.08.008 